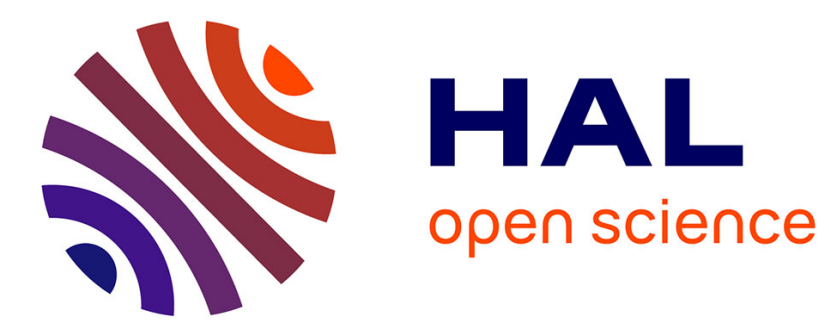

\title{
Brillouin light-scattering from polymer gels
}

S. Ng, Y. Li

\section{- To cite this version:}

S. Ng, Y. Li. Brillouin light-scattering from polymer gels. Journal de Physique II, 1993, 3 (8), pp.1241-1245. 10.1051/jp2:1993194 . jpa-00247900

\section{HAL Id: jpa-00247900 https://hal.science/jpa-00247900}

Submitted on 1 Jan 1993

HAL is a multi-disciplinary open access archive for the deposit and dissemination of scientific research documents, whether they are published or not. The documents may come from teaching and research institutions in France or abroad, or from public or private research centers.
L'archive ouverte pluridisciplinaire HAL, est destinée au dépôt et à la diffusion de documents scientifiques de niveau recherche, publiés ou non, émanant des établissements d'enseignement et de recherche français ou étrangers, des laboratoires publics ou privés. 
Classification

Physics Abstracts

$62.90-78.35-82.70$

\title{
Brillouin light-scattering from polymer gels
}

\author{
S. C. $\mathrm{Ng}$ and Y. Li \\ Department of Physics, National University of Singapore, Kent Ridge, Singapore 0511 \\ (Received 2 April 1993, accepted in final form II May 1993)
}

\begin{abstract}
The experimental results of the Brillouin shift and width for poly(vinyl alcohol) and poly(vinyl chloride) gels as a function of gel network volume fraction have been analysed using the theory of Marqusee and Deutch for Brillouin light-scattering from polymer gels, incorporated with a new plausible assumption relating the speed of sound in the gel network with its volume fraction. It has been found that the essential features of the behaviour of the Brillouin shift and width are well described by the theory over most of the range of the volume fraction.
\end{abstract}

\section{Introduction.}

A theory for Brillouin light-scattering from polymer gels was proposed by Marqusee and Deutch (MD) in 1981 [1]. Since then little attention has been focused on providing detailed experimental data for comparison. To our knowledge there have been only three detailed experimental studies [2-4] which may be analysed using the MD theory. In fact, two of these studies, which are on poly(vinyl alcohol) (PVA) hydrogels [2] and on solutions of poly(methyl methacrylate) (PMMA) in toluene [4], have already been discussed in the light of the MD theory. The third study on solutions of poly(vinyl chloride) (PVC) in acetone [3] however has only been analysed using a theory proposed by Johnson for the elastodynamics of gels [5], which contrary to expectation fails to account for the observations in the gelation system. While the gross features of the MD model agree with the experimental measurements on PVA and PMMA gels, in order to obtain a more realistic description, it was found necessary to vary the model parameters in a rather artificial manner that had little physical justification [2, 4]. In particular, the speed of the sound in the gel network must be forced to depend strongly on the network volume fraction in contradiction with the original expectation [1]. In this paper a simple plausible relationship between the speed of sound in the network and its volume fraction is proposed and new analyses on the experimental data of PVA and PVC gels based on the MD theory are presented.

\section{Summary of the MD theory incorporated with a new assumption.}

Marqusee and Deutch treat a polymer gel as a coupled fluid and network system. They define a parameter $0 \leqslant \lambda \leqslant 1$ which is a measure of the degree of coupling between elastic waves in the 
network and fluid. Two limiting cases are considered by them. The first case occurs for small frictional damping $f$ and predicts essentially a two-mode type of behaviour for the Brillouin spectrum. In this case two pairs of Brillouin peaks should be observable, which for weak coupling $\lambda \simeq 0$, are close to the Brillouin angular frequencies $\pm \omega_{0}$ and $\pm \omega_{n}$. Here $\omega_{0}=C_{0} k$ and $\omega_{\mathrm{n}}=C_{\mathrm{n}} k, C_{0}$ and $C_{\mathrm{n}}$ are respectively the speed of sound in the fluid and network and $k$ is the sound wave vector. The other limiting case is for strong frictional damping. It corresponds to a one-mode type of behaviour which predicts a single pair of Brillouin peaks at angular frequencies $\pm \bar{\omega}$. Here $\bar{\omega}=\bar{C} k$ and $\bar{C}$ is the average speed of sound in the gel. If $\phi$ is the volume fraction of the gel network, $\bar{C}(\phi=0)=C_{0}$ and $\bar{C}(\phi=1)=C_{n}$.

All measurements of Brillouin spectra of PVA, PVC as well as PMMA gels show the presence of only a single pair of modes. It therefore seems appropriate to compare these results with the theory for the limit of strong friction. Marqusee and Deutch give two equations for the $\phi$-dependence of the true Brillouin shift $\nu_{\mathrm{B}}$ and true Brillouin width $\Gamma_{\mathrm{B}}$ (FWHM):

$\nu_{\mathrm{B}}=\bar{C} k / 2 \pi$, where

$$
\bar{C}^{2}=\frac{\left.C_{0}^{2} \rho_{\mathrm{f}}+\phi\left(C_{\mathrm{n}}^{2} \rho_{\mathrm{n}}-C_{0}^{2} \rho_{\mathrm{f}}\right)+2 C_{0} C_{\mathrm{n}} \sqrt{\lambda \rho_{\mathrm{n}} \rho_{\mathrm{f}}\left(\phi-\phi^{2}\right.}\right)}{\rho_{\mathrm{f}}+\phi\left(\rho_{\mathrm{n}}-\rho_{\mathrm{f}}\right)}
$$

and $\Gamma_{\mathrm{B}}=\Gamma k^{2} / \pi$, where

$$
\begin{aligned}
2 \Gamma= & \frac{4 \eta_{\mathrm{S}} / 3+\eta_{\mathrm{B}}}{\rho_{\mathrm{f}}+\phi\left(\rho_{\mathrm{n}}-\rho_{\mathrm{f}}\right)}+\frac{\rho_{\mathrm{f}}^{2} \rho_{\mathrm{n}}^{2}\left(\phi-\phi^{2}\right)^{2}}{\left(\rho_{\mathrm{f}}+\phi\left(\rho_{\mathrm{n}}-\rho_{\mathrm{f}}\right)\right)^{3}} \frac{1}{f \bar{C}^{2}} \\
& \times\left\{C_{0}^{2}-C_{\mathrm{n}}^{2}+C_{0} C_{\mathrm{n}}\left[\rho_{\mathrm{f}}-\phi\left(\rho_{\mathrm{n}}+\rho_{\mathrm{f}}\right)\right] \sqrt{\frac{\lambda}{\rho_{\mathrm{n}} \rho_{\mathrm{f}}\left(\phi-\phi^{2}\right)}}\right\}^{2}
\end{aligned}
$$

Here $\rho_{f}$ and $\rho_{\mathrm{n}}$ are the densities of the fluid and network respectively, $\eta_{\mathrm{S}}$ and $\eta_{B}$ are respectively the shear and bulk viscosities in the fluid. Note that there is a typo error in the sign before the $C_{0} C_{\mathrm{n}}$ term in the equation (2) in reference [1]. To satisfy the criterion of a strong frictional damping, $f \gg\left(\frac{4}{3} \eta_{\mathrm{S}}+\eta_{\mathrm{B}}\right) k^{2}$ or $2 \pi \Gamma_{\mathrm{B}} \rho_{\mathrm{f}}(1-\phi)$. Originally $C_{\mathrm{n}}$ in equations (1) and (2) was assumed to be only weakly dependent of $\phi$. In comparison with experimental data, the prediction of the MD theory for $\nu_{B}$, equation [1] calculated with constant $C_{\mathrm{n}}=\bar{C}(\phi=1)$, provides an incorrect curvature of the $\nu_{\mathrm{B}}$ versus $\phi$ dependence [2, 4]. In order to obtain a realistic description of the experimental data in terms of the MD theory, it is necessary to suppose that $C_{\mathrm{n}}$ is strongly $\phi$-dependent $[2,4]$.

For PVA and PVC gels, the prepolymerized crosslinked samples were first fully swelled by water and acetone respectively $[2,3]$. The Brillouin spectrum of each sample was then measured as a function of gel network volume fraction $\phi$ at room temperature. The volume fraction $\phi$ was varied by controlled evaporation of the fluid from the gel. This corresponds to gels with fixed degree of polymerization but varying amount of solvent. According to de Gennes [6] the bulk modulus $B$ of such gels should scale like $\phi T / N$ where $T$ is the absolute temperature and $N$ the degree of polymerization. Both $T$ and $N$ are constant for the PVA and PVC gels investigated. The sound velocity $C$ is related to $B$ by the well-known equation $C=\sqrt{B / \rho}$ where $\rho$ is the gel density. In view of these, it is plausible to assume that $C_{\mathrm{n}}=C_{\mathrm{n}}(\phi)=\bar{C}(\phi=1) \sqrt{\phi}$. The new calculations for the PVA and PVC gels are based on this simple proposed relationship and the MD theory. 


\section{Results of analyses.}

Full details of the Brillouin light-scattering measurements and method of removing the instrumental response to obtain the true Brillouin shift $\nu_{\mathrm{B}}$ and width $\Gamma_{\mathrm{B}}$, as well as the refractive index measurements for both PVA and PVC gels are given in our earlier works [2, 3, 7]. The sound wave vector $k$ is determined by light-scattering geometry,

$$
k=\frac{4 \pi}{\lambda} n \sin \frac{\theta}{2}
$$

where $\lambda=514.5 \mathrm{~nm}, \theta=90^{\circ}$ and $\mathrm{n}$ is the index of refraction which is parameterized in terms of a second order polynomial in $\phi$ :

$$
n(\phi)=n_{0}+n_{1} \phi+n_{2} \phi^{2}
$$

The relevant values for the physical parameters [2, 3] required for the calculations are listed in table I. The resulting predictions for $\nu_{\mathrm{B}}$ as a function of $\phi$ in PVA and PVC gels are displayed in figure 1 for the limiting cases of weak coupling $\lambda \simeq 0$ as solid curve and maximum coupling $\lambda=1$ as broken curve. The open circles are measured values. It is evident that the predictions for the limiting case of weak coupling agree quite well with the experimental data. The incorrect curvature, reported earlier [2], of the $\nu_{\mathrm{B}}$ versus $\phi$ dependence calculated with constant $C_{\mathrm{n}}=\bar{C}(\phi=1)$ has been rectified. Figure 2 shows the predictions of the MD theory for $\Gamma_{\mathrm{B}}$ as a function of $\phi$ in PVA and PVC gels for the limiting cases of $\lambda \simeq 0$ and $\lambda=1$ using the physical parameters in table I, together with the measured values. Constant values for the frictional damping of $f(\lambda=0)=1.3 \times 10^{13}, f(\lambda=1)=$ $2.2 \times 10^{13} \mathrm{kgm}^{-3} \mathrm{~s}^{-1}$ for PVA gel and $f(\lambda \approx 0)=7.2 \times 10^{12}, \quad f(\lambda=1)=$ $2.33 \times 10^{13} \mathrm{kgm}^{-3} \mathrm{~s}^{-1}$ for PVC gel have been chosen. Here again the MD theory for $\lambda \simeq 0$ successfully predicts the essential features of $\Gamma_{\mathrm{B}}$, particularly the presence of maximum at intermediate $\phi$ value for both PVA and PVC gels. It is to be noted that the values chosen for $f$

Table I. - The bulk physical parameters of PVA and PVC at room temperature [2, 3]. All the symbols used are defined in the text.

\begin{tabular}{|c|c|c|c|}
\hline Parameter & PVA & PVC & Unit \\
\hline$\rho_{\mathrm{f}}$ & 998 & 800 & $\mathrm{~kg} \mathrm{~m}^{-3}$ \\
\hline$\rho_{\mathrm{n}}$ & 1269 & 1370 & $\mathrm{~kg} \mathrm{~m}^{-3}$ \\
\hline$C_{0}=\bar{C}(\phi=0)$ & 1475.5 & 1179 & $\mathrm{~m} \mathrm{~s}^{-1}$ \\
\hline$C_{\mathrm{n}}=\bar{C}(\phi=1)$ & 3783.6 & 2418 & $\mathrm{~m} \mathrm{~s}^{-1}$ \\
\hline$\frac{4}{3} \eta_{\mathrm{S}}+\eta_{\mathrm{B}}$ & $2.92 \times 10^{-3}$ & $2.66 \times 10^{-3}$ & $\mathrm{~kg} \mathrm{~m}^{-1} \mathrm{~s}^{-1}$ \\
\hline$n_{0}$ & 1.3365 & 1.3679 & \\
\hline$n_{1}$ & $1.0648 \times 10^{-1}$ & $1.709 \times 10^{-1}$ & \\
\hline$n_{2}$ & $6.6895 \times 10^{-2}$ & 0 & \\
\hline
\end{tabular}




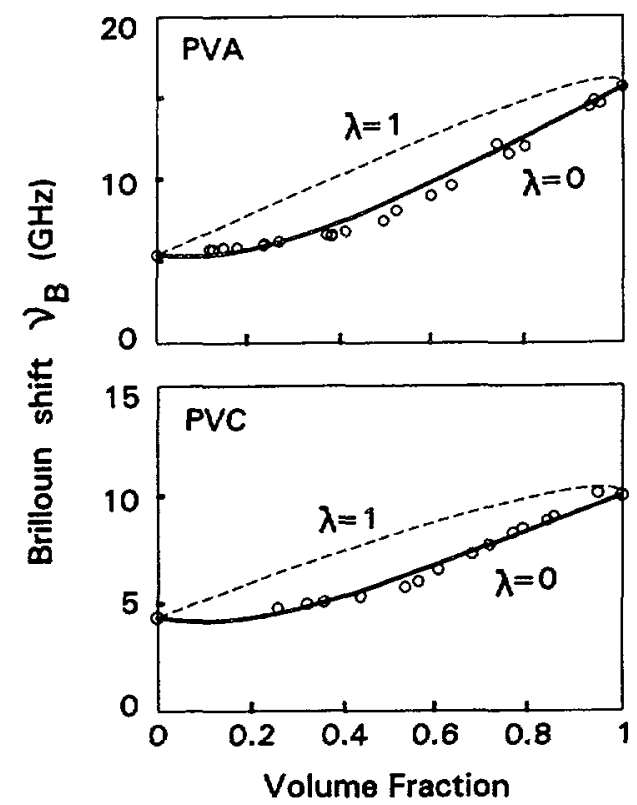

Fig. 1.

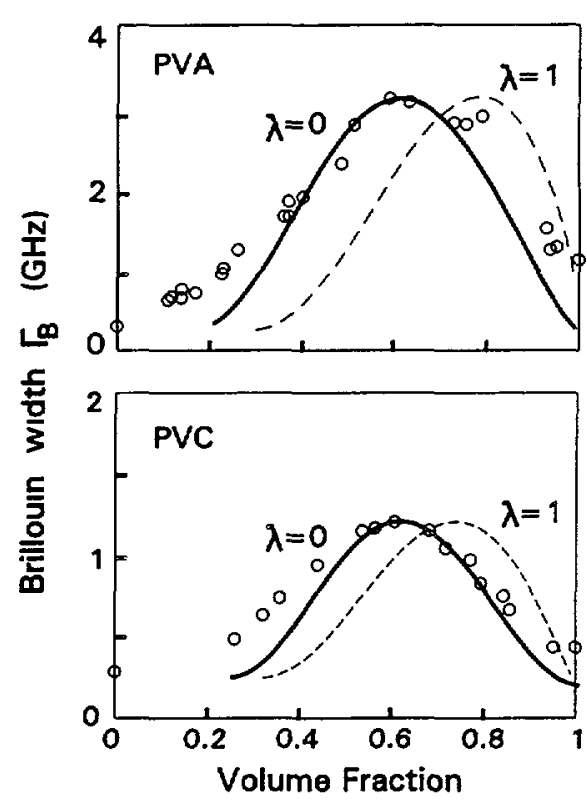

Fig. 2.

Fig. 1. - The Brillouin shift in PVA and PVC gels as a function of network volume fraction. Open circles are experimental data. Solid and broken lines are theoretical curves for $\lambda \simeq 0$ and $\lambda=1$ respectively (See text).

Fig. 2. - The Brillouin width in PVA and PVC gels as a function of network volume fraction. Open circles are experimental data. Solid and broken lines are theoretical curves for $\lambda \simeq 0$ and $\lambda=1$ respectively (See text).

to produce the calculated results for $\Gamma_{\mathrm{B}}$ satisfy fairly well the criterion for a strong frictional damping.

\section{Conclusion.}

Previous measurements of the dependence of the true Brillouin shift $\nu_{B}$ and width $\Gamma_{\mathrm{B}}$ on the volume fraction $\phi$ of the gel network for PVA and PVC gels have been compared with the theory of Marqusee and Deutch for Brillouin light-scattering from polymer gels, incorporated with a new assumption regarding the relationship between the speed of the sound in the gel network and its volume fraction, $C_{\mathrm{n}}(\phi)=\bar{C}(\phi=1) \sqrt{\phi}$. It is found that, even with such a simplistic assumption, the measurements of $\nu_{\mathrm{B}}$ and $\Gamma_{\mathrm{B}}$, over most of the range of $\phi$ are amazingly consistent with the essential features of the predictions of the MD theory for the case of weak coupling $(\lambda=0)$ and strong frictional damping $f$. In fact, the agreement between theory and experiment for $\nu_{\mathrm{B}}$ over the entire range of $\phi$ is good. There are, however, deviations for $\Gamma_{B}$ at small and high volume fractions which may imply that a more refined theory needs to be developed. Analysis of the PMMA gels at low volume fraction $(0.1 \leqslant \phi \leqslant 0.5)$ by Brown et al. [4] using the MD theory suggests that the bulk modulus of their gels scales as $\phi^{26}$ which is close to $\phi^{225}$ predicted by the blob theory [6]. It is to be noted, however, that their sample preparation is very different from that of PVA and PVC gels. In 
particular, the degree of the polymerization of their gels is $\phi$ dependent, whereas that of PVA and PVC gels is a $\phi$-independent constant. This is why the bulk modulus of our gels has been chosen to scale as $\phi$ in our analysis. In conclusion, the results of the present paper have clearly demonstrated the usefulness of the theory of Marqusee and Deutch for Brillouin lightscattering from polymer gels in offering a realistic description of the $\phi$-dependence of $\nu_{\mathrm{B}}$ and $\Gamma_{\mathrm{B}}$, provided that a relationship between $C_{\mathrm{n}}$ and $\phi$, appropriate to the gel sample preparation, is incorporated into the theory.

\section{References}

[1] Marqusee J. A., Deutch J. M., J. Chem. Phys. 75 (1981) 5239.

[2] Ng S. C., Hosea T. J. C., Gan L. M., J. Phys. France Lett. 46 (1985) L-887.

[3] Ng S. C., Hosea T. J. C., Teoh S. H., Chem. Phys. 110 (1986) 225.

[4] Brown W., Schillen K., Johnsen R., Konak C., Dvoranek L., Macromolecules 25 (1992) 802.

[5] Johnson D. L., J. Chem. Phys. 77 (1982) 1531.

[6] De Gennes P. G., Scaling Concepts in Polymer Physics (Cornell University Press, 1979).

[7] Ng S. C., Hosea T. J. C., Gan L. M., Phys. Lett. A 105 (1984) 153. 